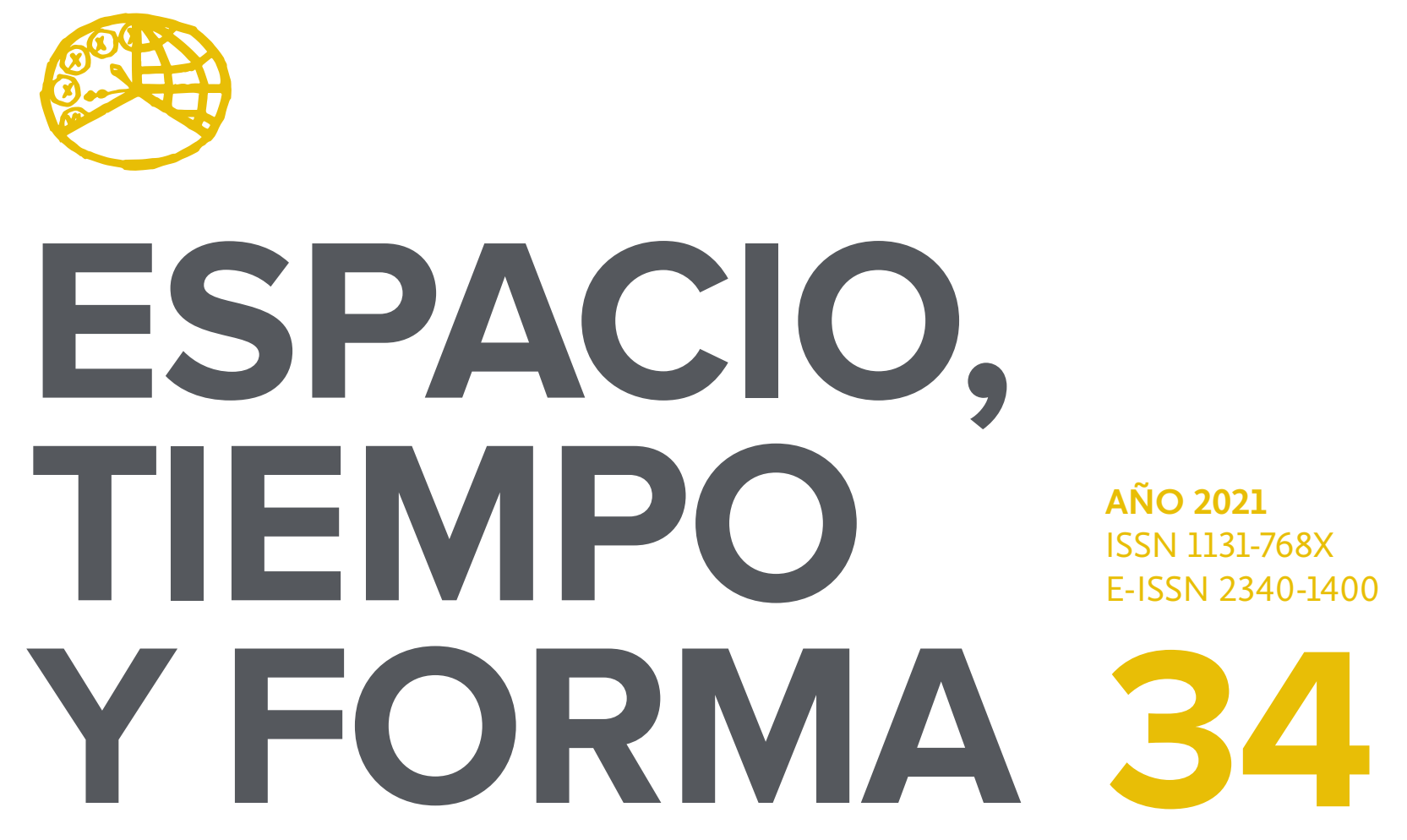

SERIE IV HISTORIA MODERNA

REVISTA DE LA FACULTAD DE GEOGRAFÍA E HISTORIA

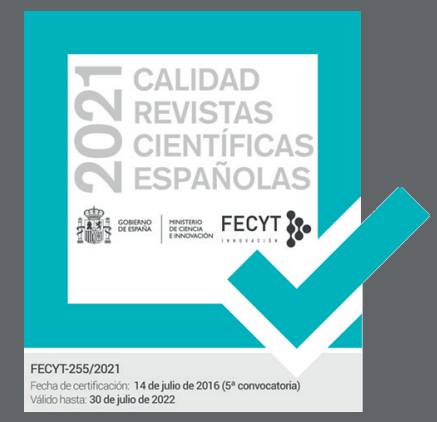




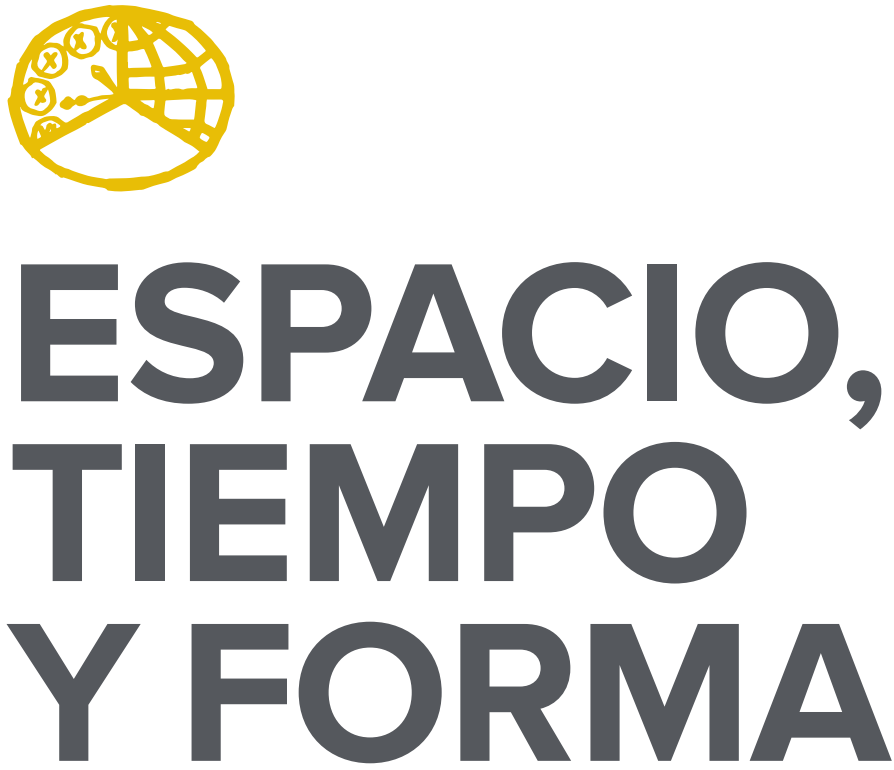

AÑO 2021

ISSN 1131-768X

E-ISSN 2340-1400

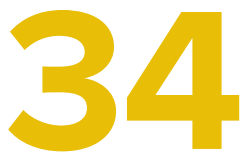

SERIE IV HISTORIA MODERNA

REVISTA DE LA FACULTAD DE GEOGRAFÍA E HISTORIA

DOI: https://doi.org/10.5944/etfiv.34.2021

\section{UกED}

UNIVERSIDAD NACIONAL DE EDUCACIÓN A DISTANCIA 
La revista Espacio, Tiempo y Forma (siglas recomendadas: ETF), de la Facultad de Geografía e Historia de la UNED, que inició su publicación el año 1988, está organizada de la siguiente forma:

$$
\begin{aligned}
& \text { SERIE I - Prehistoria y Arqueología } \\
& \text { SERIE II - Historia Antigua } \\
& \text { SERIE III - Historia Medieval } \\
& \text { SERIE IV - Historia Moderna } \\
& \text { SERIE V - Historia Contemporánea } \\
& \text { SERIE VI - Geografía } \\
& \text { SERIE VII - Historia del Arte }
\end{aligned}
$$

Excepcionalmente, algunos volúmenes del año 1988 atienden a la siguiente numeración:

$$
\begin{aligned}
& \mathrm{N} .^{\circ} 1 \text { - Historia Contemporánea } \\
& \mathrm{N}^{\circ} 2 \text { - Historia del Arte } \\
& \mathrm{N} .^{\circ} 3 \text { - Geografía } \\
& \mathrm{N} .^{\circ} 4 \text { - Historia Moderna }
\end{aligned}
$$

ETF no se solidariza necesariamente con las opiniones expresadas por los autores.

\author{
UNIVERSIDAD NACIONAL DE EDUCACIÓN A DISTANCIA \\ Madrid, 2021 \\ SERIE IV · HISTORIA MODERNA N. ${ }^{\circ} 34,2021$ \\ ISSN $1131-768 X \cdot$ E-ISSN 2340-1400 \\ DEPÓSITO LEGAL \\ M-21.037-1988 \\ URL \\ ETF IV · HISTORIA MODERNA · http://revistas.uned.es/index.php/ETFIV \\ DISEÑO Y COMPOSICIÓN \\ Carmen Chincoa Gallardo · http://www.laurisilva.net/cch \\ Impreso en España · Printed in Spain
}

(c) (7) (8) Esta obra está bajo una licencia Creative Commons Reconocimiento-NoComercial 4.0 Internacional. 


\section{MONOGRÁFICO · SPECIAL ISSUE}

LA POLÍTICA ULTRAMARINA DE LAS MONARQUÍAS

IBÉRICAS (CIRCA 1700-1750): UNA HISTORIA

DE FRACASOS Y ÉXITOS RELATIVOS

THE OVERSEAS POLICY OF THE IBERIAN MONARCHIES (CIRCA 1700-1750): A HISTORY OF FAILURES AND RELATIVE SUCCESSES 



\title{
EL FRACASO DE LOS PROYECTOS DE REFORMA EN EL VIRREINATO PERUANO DE PRINCIPIOS DEL SIGLO XVIII. LAS PROPUESTAS DE CARMINE NICOLA CARACCIOLO, PRÍNCIPE DE SANTOBUONO
}

\section{THE FAILURE OF REFORM PROJECTS IN \\ THE PERUVIAN VICEROYALTY AT THE BEGINNING OF THE EIGHTEENTH CENTURY. THE PROPOSALS OF CARMINE NICOLA CARACCIOLO, PRINCE OF SANTOBUONO}

\author{
Valentina Favarò ${ }^{1}$ \\ Recibido: 15/12/2020 - Aceptado: 22/04/2021 \\ DOI: https://doi.org/10.5944/etfiv.34.2021.29357
}

\begin{abstract}
Resumen ${ }^{2}$
Desde hace algunas décadas, una nueva corriente historiográfica ha dirigido su atención a las transformaciones que, en los ámbitos social, político y militar, impulsó el gobierno borbónico en las primeras décadas del siglo XVIII. Aunque en gran medida no tuvieron éxito, las reformas promovidas en las dos primeras décadas entre la Península Ibérica, Sicilia, Nueva España y Perú ponen de relieve un importante primer intento de hacer más funcional el gobierno de la Monarquía. Este artículo tiene como objetivo analizar las propuestas de las reformas que Carmine Nicola Caracciolo elaboró durante su mandato como Virrey del Perú (I7I6-I72I). En particular, se reflexionará sobre aquellas propuestas que no dieron lugar a un resultado positivo: la creación del Virrey de Nueva Granada; la propuesta de reforma de los servicios postales; la propuesta de abolir el sistema de la mita; la revisión del papel de los corregidores.
\end{abstract}

Palabras clave

Siglo XVIII; Monarquía de España; reformas; Carmine Nicola Caracciolo

\footnotetext{
1. Università degli Studi di Palermo; valentina.favaro@unipa.it

2. Este estudio forma parte del proyecto Reloj de Indias: la proyección europea en el Atlántico, 1665-1700 (Sl1) PJI/2019-00270), concedido por la Comunidad de Madrid y la Universidad Autónoma de Madrid, y también se vincula a los resultados del proyecto Práctica de gobierno y cultura política: Europa y América en la monarquía de España, 1668-1725 [PID2019-108822GB-loo], concedido por el Ministerio de Ciencia e Innovación.

Abreviaturas utilizadas: Archivio di Stato di Napoli (ASN, Nápoles), Archivo General de Indias (AGI, Sevilla), Archivo Histórico Nacional de Quito (AHNQ, Quito), Real Biblioteca (RB, Madrid).
} 


\begin{abstract}
For a some decades, a new historiographical current has turned its attention to the transformations that, in the social, political and military spheres, prompted the Bourbon government in the early decades of the eighteenth century. Although largely unsuccessful, the reforms promoted in the first two decades between the Iberian Peninsula, Sicily, New Spain and Peru highlight an important first attempt to make the monarchy government more functional. This article aims to analyse the proposals for the reforms that Carmine Nicola Caracciolo developed during his tenure as Viceroy of Peru (I716-I72I). In particular, we will reflect on those proposals that did not lead to a positive result: the creation of the viceroyalty of New Granada; the proposed reform of postal services; the proposal to abolish the mita; reviewing the role of corregidores.
\end{abstract}

\title{
Keywords
}

I ${ }^{\text {th }}$ Century; Spanish Monarchy; Reforms; Carmine Nicola Caracciolo 
DESDE HACE ALGUNAS DÉCADAS, una nueva corriente historiográfica ha dirigido su atención a las transformaciones que, en los ámbitos social, político y militar, impulsó el gobierno borbónico en las primeras décadas del siglo XVIII. Superada la idea de que la fase reformista estaba estrictamente anclada a los reinados de Carlos IIl y Fernando VI, se destacó cómo -aunque a veces de manera embrionaria o incompleta- ya en los años de Felipe $\mathrm{V}$ se habían promovido intervenciones que interesaban las distintas áreas administrativas.

Así, por un lado, en el contexto más amplio de la monarquía española, desde la realidad peninsular hasta la colonial, la intención de hacer más funcional la máquina administrativa contribuyó a modificar la planta de gobierno; por el otro, destacó todas las dificultades inherentes a los proyectos de reforma. Esta perspectiva, más orientada a captar los fracasos que a los procesos de cambios en las estructuras políticas y administrativas finalmente ejecutados, es particularmente interesante, pues permite reflexionar sobre algunos temas cruciales. Lejos de ser lineales y «neutrales» las reformas en su elaboración y en los objetivos preliminares, aquéllas se convierten en expresión de equilibrios a redefinir, de juegos de poder, de la necesidad de crear nuevas jerarquías dentro de la corte o en contextos locales. Analizar su fracaso, por tanto, posibilita sacar a la luz la complejidad de estas dinámicas, los resultados inesperados, las resistencias levantadas por aquellos actores que en tales reformas percibieron un daño potencial a sus propios privilegios.

Para comprobar estas afirmaciones, en el presente artículo se ha optado por utilizar, como case study, el corpus de reformas que Carmine Nicola Caracciolo, príncipe de Santobuono, presentó al Consejo de Indias durante los años de su virreinato en el Perú (I7I6-I72I). Se trata de intervenciones que, impulsadas en algunos casos por el cardenal Giulio Alberoni, deberían haber contribuido -según su elaboración teórica- a hacer más eficiente la gestión del «gobierno a distancia» y a eliminar las prácticas ilícitas que afectaban a los reinos del Perú. Una mirada más profunda, sin embargo, muestra cómo la definición de las reformas estaba fuertemente conectada con intereses personales y faccionales que entrelazaban a Lima y Madrid, entre las élites locales y los ministros en la corte. Por lo tanto, comprender su fracaso significa comprender las jerarquías y la fluidez de las relaciones de poder, y no solo la existencia o no de una planificación madura.

\section{CARMINE NICOLA CARACCIOLO: EL HOMBRE, LA POLÍTICA Y LA CORTE}

La adopción de este punto de observación requiere una breve reconstrucción del perfil del virrey y del contexto en el que comenzó y se consolidó gradualmente su cursus honorum. Trazar los contornos del marco político en el que se insertó su actividad es funcional, de hecho, para sustentar la interpretación adoptada: la conexión entre las opiniones negativas sobre las reformas de Caracciolo pronunciadas por el Consejo de Indias y la expulsión del cardenal Giulio Alberoni desde la corte. La redefinición del equilibrio de poder en el que se involucró directamente el príncipe de Santobuono fue un elemento determinante -aunque, obviamente, no el único-para 
sancionar el fracaso de su intento de realizar cambios significativos en algunos ámbitos administrativos y gubernamentales.

Miembro de una de las familias aristocráticas más importantes del reino de Nápoles, embajador felipista ante la Santa Sede y la República de Venecia durante la guerra de Sucesión española ${ }^{3}$, Carmine Nicola Caracciolo fue el primer italiano en obtener un nombramiento para el virreinato andino. En los años del reinado de Felipe V, la atribución de oficios y honores a exponentes de élite de la península Itálica, antes -en gran medida- reservada a un grupo más reducido de Grandes o Títulos castellanos o de la Corona de Aragón, fue una de las herramientas más importantes para fortalecer la fidelidad al soberano. No es casual, por tanto, que en esta particular situación el cargo virreinal fuera atribuido a Caracciolo.

En los años vividos en la península Ibérica -especialmente en Madrid, pero también en Cádiz y Alicante- tanto en un plano más circunscrito y familiar, como en uno más amplio y faccional, los vínculos que don Carmine Nicola estrechó fortalecieron la red de poder desde la que tomó ventaja. En el primer caso, desempeñó un papel importante su esposa, Costanza Ruffo, nombrada dama de la reina María Luisa Gabriela de Saboya en I7ı2, y su hija Clara Irene quien, ese mismo año, se casó con el marqués de Crevacuore 4 . Este último, heredero de los príncipes de Masserano, fue considerado uno de los referentes más importantes dentro de la corte de Felipe $\mathrm{V}$, capaz de ejercer una fuerte influencia sobre la soberana. En un nivel más amplio, además, Caracciolo disfrutó de la mayor participación de la nobleza «italiana» en papeles clave de la administración, resultado de la necesidad del nuevo soberano de consolidar las prácticas de lealtad y evitar el riesgo de que las élites locales apoyaran a la facción austriaca. Unos años más tarde, esta implicación se vio reforzada tras algunos acontecimientos, como la boda de Felipe V y Elisabetta Farnese y la llegada a Madrid del futuro cardenal Giulio Alberoni.

De hecho, la presencia de la nueva reina y del cardenal cambió el equilibrio faccional y dio lugar a una línea política que poco a poco iría ensombreciendo al partido francés y fortaleciendo a aquellos exponentes de la aristocracia italiana que, durante años, habían girado en torno a la figura del soberano: a partir de ese momento, Giulio Alberoni, el duque de Popoli y los príncipes de Giovinazzo, Cellammare y Santobuono constituyeron el grupo más sólido y cohesionado del partido italiano en la corte, capaz de sobrevivir a la purga iniciada en la segunda década del siglo XVIII5. Además de la princesa Orsini, expulsada inmediatamente de España por Elisabetta Farnese a su llegada, otros ministros cercanos a ella tuvieron que dejar sus cargos y abandonar Madrid. Jean Orry, consejero de la Real Hacienda, fue destituido de su cargo en febrero de I7I5 y el mismo día fue desterrado el jurista Melchor Rafael de Macanaz, mientras que el confesor real Pierre Robinet fue sustituido por el jesuita Daubenton.

\footnotetext{
3. FAVARÒ, 2018.

4. Además de Costanza Ruffo, la princesa de Atri, la duquesa de Havré - de la familia Lanti della Rovere-y la princesa de Robecq también fueron damas de María Luisa. LóPEZ-CORDóN, 2008: 1382-1383.

5. Restaino Cantelmo, VIII duque de Popoli, obtuvo el título de grande de España en 1702 y había sido nombrado capitán de la Compañía Italiana de la nueva Guardia de Corps del soberano; en 1713 comandó las tropas borbónicas enviadas a Barcelona y al año siguiente obtuvo el Toisón de Oro. ANdúJAR CASTILLO, 2008: 112-117.
} 
El objetivo era socavar el peso político y la injerencia de Versalles, a la que también contribuiría la muerte, el I de septiembre de I7I5, del rey Luis XIV.

Caracciolo se convirtió en una parte estratégica de este grupo faccional italiano, especialmente tras el estrecho vínculo con Giulio Alberoni ${ }^{6}$, quien pronto fue el principal interlocutor de la reina Elisabetta Fernese ${ }^{7}$. Compartir la línea política del abate parmesano y participar en la planificación de una nueva forma de gobierno - orientada a privilegiar la vía reservada para fortalecer, en primer lugar, el propio poder y, en segundo, la nueva Secretaría del Despacho Universal de Asuntos de Indias a expensas de las prerrogativas y funciones hasta entonces asumidas por el Consejo de Indias ${ }^{8}$ - hizo que Caracciolo siguiera su destino, desde su ascenso hasta su rápido declive. Y de esto dependió también el resultado de las reformas propuestas, desde la creación del virreinato de Nueva Granada, al sector minero, al sistema postal, a la recaudación de la alcabala, al monopolio del tabaco y del papel y, en sí, a la revisión de la organización de la Real Hacienda.

\section{EL VIRREINATO DE NUEVA GRANADA}

El Príncipe de Santobuono llegó a Cartagena de Indias en enero de i7i6 y permaneció allí unos meses, antes de continuar su viaje hacia la Ciudad de Los Reyes. La estancia en Cartagena, junto al gobernador y los oficiales reales de esa provincia, permitió a Carmine Nicola Caracciolo adquirir informaciones relativas al funcionamiento del virreinato y empezar a reflexionar sobre las políticas a adoptar para lograr los objetivos personales y gubernamentales ${ }^{9}$. En este contexto, se puede leer el compromiso de

6. Existe una extensa bibliografía sobre el cardenal Giulio Alberoni, que incluye MAQUEDA ABREU, 2009; Martínez Navas, 2010; Alabrús Iglesias, 2011; y Barrio Gozalo, 2011.

7. Sobre la figura de Elisabetta Farnese y su papel en el marco político internacional, véase FrAGNITO, 2009; MAFrICI, 2019; SOdANO, 2011; y VÁZQUez GESTAL, 2013.

8. El uso de la vía reservada y el agotamiento de las prerrogativas del Consejo de Indias formaban parte de diversas medidas del reformismo borbónico y, sobre todo, respondían a la voluntad de Giulio Alberoni de limitar sus ámbitos de actuación. El purpurado identificó en los miembros del Consejo, de hecho, a algunos responsables de las continuas violaciones a las normas que regulaban las relaciones con las Indias. En una carta a Caracciolo, escribió que reduciría el número de miembros a cinco o seis oficiales y que «si vede che il governo nelle Indie è il più ingiusto e il più scellerato che sia al mondo (...) si è veduto un commercio infame e scandaloso, che si fa in questi consiglieri (...) e suppongo che alcuni verrano privati del loro impiego». ASN, Archivio privato Santobuono, vol. 40, f. 3, n.n., Madrid, Carta del cardinal Alberoni a el principe de Santobuono, Madrid 19 octubre 1716. En varias ocasiones se pidió al príncipe de Santobuono que se comunicara directamente con el soberano, sin la intermediación del Consejo. Ver, por ejemplo, AHNQ, Corte Suprema, Cedularios, caja 7, c. 156. En el mismo 1717 Alberoni había ordenado el traslado de la Casa de Contratación y el Consulado de Cargadores de Indias de Sevilla a Cádiz. Cfr. KuETHE, 2013; CRESPO SolANA, 1996.

9. La parada de los virreyes entre Panamá y Cartagena era obligatoria y se incluía en uno de los puntos de las instrucciones emitidas por el soberano. En el caso del príncipe de Santobuono, en la Instruccion que de orden de S.M. se han mandado formar para que el Signor virrey principe di Santobono la tenga presente y puntualmente la observe, se especificó que «llegado el señor virrey a Portobelo visitará por su persona los castillos de la Gloria, el de Todo Fierro y el de San Gerónimo, mandando pasar muestra de todas placas de su dotaciones y por su persona a reconocimiento de sus armas, artilleria y municiones y ynformado de estar o no completo (...) con la misma reserba ynformará a S. M. y la causa en caso de no estar completo. Y pues el presidente de Panamá ha de baxar a Portovelo al recivimiento del señor virrey, combendrá mucho que S. E. con dicho presidente vean y confieran el modo de ympedir la franqueza con que ingleses y olandeses mantienen continuado el comercio en el paraje de aquella costa que llaman de Vastimentos, a mui corta distancia da la ciudad de Portobelo». ASN, Archivio privato Santobuono, vol. 39. Madrid 20 diciembre 1711. 
Caracciolo de implementar las reformas propuestas por Felipe $V$ destinadas a lograr una mayor eficiencia en el funcionamiento de la máquina burocrática. De hecho, la acción de Santobuono fue una expresión de la clara determinación del soberano de rediseñar el plan de gobierno para resolver las necesidades financieras y administrativas y reducir mal gobierno y prácticas ilícitas. Necesidad que se sintió aún más en los territorios del Nuevo Mundo, donde la distancia del corazón palpitante de la Monarquía favoreció la adquisición de mayores poderes por parte de sus representantes ${ }^{\mathrm{T}}$.

La distancia del soberano -y de su control- hizo que los oficiales aumentaran sus competencias, determinando así también una posible divergencia entre las líneas políticas defendidas por Madrid y lo que efectivamente se promovía a nivel local. Además, la presencia de múltiples poderes fuertemente arraigados en la sociedad peruana, hizo aún más complicado llevar a cabo una práctica de gobierno eficaz. En este contexto, en marzo de I7I6, Caracciolo formuló la propuesta de creación de un tercer virreinato que debía incluir las regiones entre Guayana, el istmo de Panamá y el reino de Quito ${ }^{\text {II }}$. La propuesta fue legitimada por la necesidad de contener las dinámicas políticas corruptas e ineficientes del arco caribeño, zona del virreinato peruano que sufría las limitaciones gestoras derivadas de la distancia tanto de Madrid como de Lima.

Caracciolo -en la correspondencia que mantuvo con el soberano y Giulio Alberoniexpuso su propia evaluación, que fue aceptada por ambos interlocutores sin que se hiciera partícipe al Consejo de Indias. Como señala Francisco Eissa-Barroso, en su volumen dedicado a la fundación del virreinato, «there is no record of consulta produced by the Council of the Indies», el cual «did not receive officially notification that a new viceroyalty had been created until almost 18 months after the event ${ }^{12}$. Esta centralización siguió siendo propia de la administración del reino de Nueva Granada, como se puede observar con la correspondencia realizada entre i717 y i7ig. Además, también el nombramiento del gobernador y capitán general antes, Antonio de la Pedrosa y Guerrero, y a posteriori del virrey Jordi de Vilallonga (o Jorge de Villalonga), fueron fruto de los lazos personales que tanto se jactaban con el cardenal Alberoni como con el príncipe de Santobuono ${ }^{\mathrm{I} 3}$.

El procedimiento adoptado fue, evidentemente, resultado de la reforma impulsada en I7I7, mediante la cual el Consejo de Indias fue, de hecho, privado del derecho a resolver las cuestiones relativas al gobierno de los reinos americanos, tal como había sido promulgado por la cedula real de I7 de mayo de I564. Felipe Il había establecido

10. Puente Brunke, 2012.

11. La propuesta fue aceptada tanto por Alberoni como por Felipe V, quien elogió «la prova di probità, di disinteresse, d'amor e di zelo del Santo Buono verso il re e la regina» y se comprometió a evaluar la posibilidad de enviar «quanto prima un viceré che avrà la sua residenza a Santa Fe». ASN, Archivio privato Santobuono, vol. 40, f. 3, n.n., Giulio Alberoni al príncipe de Santo Buono, Madrid, 27 noviembre 1716.

12. EISSA-BARROSO, 2016: 18 y 113. El Autor también señala que «the king's ministers advocated a program of reforms both in Spain and Spanish America that aimed at establishing a more executive, effective system of monarchical government in which the king's main responsibility was no longer the provision of justice, mediation, or resolution of disputes, but rather the more active one of providing good economic government and the conditions necessary for development» (ivi, p. 18). Cfr. también BECKER y RIVAS GroOT, 1921: 200-203; TAU ANZOTAEGUI, 2004.

13. ASN, Archivio privato Santobuono, vol. 40, f. 1, c. 272, Sopra lo stabilimento del viceré nella città di Santa Fee, Lima, 14 septiembre 1719. 
que todas las órdenes generales enviadas a las Indias Occidentales eran «señaladas y [...] firmadas de los de nuestro Consejo Real de las Indias» ${ }^{\top 4}$, que gozaba del privilegio exclusivo en la comunicación con el Nuevo Mundo. Las reformas de I7I7 eliminaron esta restricción al autorizar legalmente al rey y sus ministros a comunicarse con las autoridades indígenas sin la intervención del Consejo.

En esta compleja situación de redefinición de papeles y prácticas de gobierno, la creación del nuevo virreinato fue la respuesta a los problemas locales que se percibían en la Corte, a la vez que los tiempos y la forma en que tuvo lugar dicha fundación fueron una expresión directa del conflicto que surgió entre los partidarios del control directo de la Corona sobre sus territorios y el Consejo de Indias. En I723 se abolió el virreinato y se restableció la estructura de gobierno anterior a I717. El incumplimiento de los objetivos que habían formado la base de su formación -racionalización del gobierno, mayor control de las costas, eliminación contrabando- $y$, al mismo tiempo, la ampliación de conflictos jurisdiccionales y confusión administrativa, demostraron el incumplimiento de la disposición.

La creación y asignación de nuevos cargos en un contexto fuertemente marcado por la oposición de grupos sociales, y la difícil definición de sus respectivas competencias complicaron el ejercicio del poder a nivel local. En definitiva, «the cédula abolishing the viceroyalty, dated November 5, I723, noted that a president could govern the provinces as well as a viceroy; that the viceroyalty had neither increased royal revenues nor eliminated fraud; and that it brought about a great increase in the costs of government $»^{15}$. Además, no se puede descartar que la decisión de suprimir el virreinato -que sería nuevamente instituido a partir de I739- fuera el resultado de «of general repugnance toward initiatives associated with Alberoni that prevailed in Madrid during the years following his downfall in December I719» ${ }^{16}$.

La caída de Alberoni significó el fracaso de casi todo su programa de reformas. En poco tiempo se depuró a todos los hombres de su círculo y, por tanto, se abandonaron las líneas de su política internacional. Dentro de la Monarquía borbónica, los Consejos tuvieron la oportunidad de restaurar funciones antiguas y el número de Secretarías de Estado retornaría nuevamente a cuatro. La recuperación de las plenas funciones del Consejo de Indias se viviría más gradualmente, volviendo a asumir un papel central en la gestión del gobierno de los territorios americanos ${ }^{17}$.

La hipótesis de una oposición contra el cardenal y, en consecuencia, de todos los que formaban parte de su séquito podría ser una clave interesante para interpretar las hostilidades que, a partir de I7I9, el Consejo de Indias comenzó a mostrar al príncipe de Santobuono: los proyectos que presentó fueron rechazados casi por completo y el proceso de residencia en su contra se prolongó inusualmente más allá del tiempo establecido. Evidentemente, esto no excluye que la no aceptación de las propuestas

14. Recopilación, ley 23 , título $1^{\circ}$, libro $2^{\circ}$.

15. PeARCE, 2014: 92.

16. Ibidem, p. 93.

17. «Para diciembre de 1720 había caído el tesorero general y en febrero de 1721 la tesorería volvió a su antigua planta. El 21 de marzo de 1721 el Consejo de Castilla logró la supresión de los intendentes que Alberoni había reintroducido en 1718, lo que representaba una enorme victoria pues con el regreso de los corregidores el Consejo recuperaba un mayor control sobre el gobierno provincial de la Península». EISSA-BARROSO, 2012: 304. 
estuviera determinada por su insostenibilidad objetiva. Cabe destacar también que el paso interino del cargo virreinal de Carmine Nicola Caracciolo al prelado Diego Morcillo implicó un nuevo cambio en la línea política conducida en el virreinato peruano y la definición de dinámicas que se movieron en sentido contrario a lo trazado con anterioridad ${ }^{18}$.

\section{EL CORPUS DE PROYECTOS DE 1719}

Las propuestas de reforma presentadas por Caracciolo se referían, principalmente, a la organización del trabajo en el extenso virreinato peruano. En primer lugar, el pro rex creía que la creación de gremios podría hacer más fluidas las comunicaciones entre virreyes, oficiales locales y mineros ${ }^{19}$. La propuesta surgió de los buenos resultados que había tenido la creación de dichas corporaciones en Potosí, Cailloma y Huencavelica y, por tanto, se esperaba que lo mismo pudiera ocurrir en Lipes, Aullagas, Oruño, Puno y San Antonio de Esquilache. Al mismo tiempo, empezó a madurar en el virrey la convicción de que la naturaleza de la mano de obra indígena debía ser cambiada profundamente, transformándola de servil a asalariada. Durante algún tiempo, tanto en España como en el Nuevo Mundo, se había producido un animado debate sobre la oportunidad de mantener el sistema de la mita o, más bien, de recurrir a la mano de obra libre y remunerada, posiblemente apoyada por el trabajo de condenados y vagabundos. La particular atención que el príncipe de Santobuono dedicó a la cuestión y su clara posición a favor del trabajo libre fueron, sin duda, también el resultado de su formación y del contexto social y cultural en el que se formó. La participación del aristócrata partenopeo en las actividades de las academias y círculos intelectuales de Italia, en las que se difundieron las primeras ideas preilustradas, así como el conocimiento de los debates más recientes -alimentados por juristas- sobre la ineficiencia de la mano de obra servil, constituyeron el humus sobre el que tenían fundamento las propuestas del virrey ${ }^{20}$.

En I7I8, el Consejo de Indias, con cedula del 4 de mayo, expresó una opinión favorable sobre la propuesta hecha por Carmine Nicola Caracciolo de proceder con la abolición de la mita, pero con referencia únicamente a las minas de Potosí. Sólo más tarde se formuló una opinión positiva para la aplicación de la disposición a las demás minas del reino, comenzando por Huencavelica. La oposición, sin embargo, vino en poco tiempo de la élite política local. En la metrópoli de Lima fue Dionisio de Alcedo y Herrera, ex-secretario del virrey Diego Ladrón de Guevara y ordenador del Tribunal de Cuentas, quien expresó una opinión negativa motivada sobre todo por la impopularidad que la ordenanza y los desórdenes que podrían haber surgido.

18. Para una reconstrucción más amplia de las dinámicas políticas en el virreinato del Perú durante las primeras décadas del siglo XVIII, vid. Favarò, 2019.

19. ASN, Archivio privato Santobuono, vol. 38, f. 11. Relazione sullo stato delle miniere, sin fecha. Dentro del informe también hay una reflexión sobre tres posibles medidas: incrementar el valor de la plata, «dándole el que rende entre las naciones»; recalcular a la baja, de un quinto a un décimo, el porcentaje del impuesto pagado sobre el valor de los metales preciosos; y limitar el coste del mercurio.

20. Meza Villalobos, 1976. 
La misma posición fue adoptada en I720 por el oidor de Lima y antiguo gobernador general de Chile, José de Santiago Concha, marqués de Casa Concha, a quien se había encomendado una visita a las minas del reino para evaluar, junto con el príncipe de Santobuono, la posibilidad de suspender cualquier tipo de trabajo forzoso ${ }^{21}$. La cuestión, que permanecía sustancialmente sin solución en muchas de las minas del reino ${ }^{22}$, se retomó más tarde, cuando, a través de una cedula emitida el 22 de mayo de I732, se estableció que donde aún estuviera presente, la mita no fuese abolida. La única concesión fue el aumento del salario de los mitayos a cuatro reales por día ${ }^{23}$.

Si bien el complejo proceso de volver a regular la fuerza laboral no alcanzó el objetivo deseado, constituye un importante experimento de reforma que debe leerse en un contexto más amplio. Lejos de ser el único ámbito en el que el príncipe de Santobuono intentó desarrollar propuestas concretas para el aparato administrativo indiano, la propuesta de abolición de la mita constituye una pieza de un puzzle que puede reconstruirse a través del análisis de proyectos implementados por el Consejo de Indias en diciembre de I7I9. La heterogeneidad de los problemas abordados y el esfuerzo por identificar soluciones que pudieran ser aceptadas tanto por el soberano como por las élites locales ofrecen una herramienta más para comprender la interacción entre la dimensión personal de experiencia virreinal, contexto político y social del reino e intereses de la Corona. Por tanto, el análisis de los proyectos no es útil para resaltar la implementación efectiva de las reformas en el contexto peruano, sino para entender qué conocimientos había adquirido el virrey y en qué áreas creía que fuera más importante intervenir para responder a intereses personales y apoyar la voluntad de la Corona de identificar instrumentos válidos para el aumento de la Real Hacienda ${ }^{24}$. Es en esta perspectiva, por tanto, donde radica la relevancia del análisis de todas sus propuestas.

No es sorprendente que una de las formulaciones de reforma más detalladas se refiera al suministro de mercurio y que, de hecho, sea complementaria a la propuesta de abolición de la mita. Ante las dificultades encontradas en la reorganización de las minas de Huencavelica -en las que la corrupción de los corregidores y los abusos perpetrados contra los indígenas comprometieron su funcionamiento- el príncipe de Santobuono propuso su cierre a la par que explicaba las ventajas que se podrían conseguir importando mercurio de la metrópoli mediante cuatro puntos fundamentales: detener el declive demográfico vinculado a la alta tasa de mortalidad

21. AGI, Audiencia Lima, legajo 469. Relación del estado que ha tenido, y tiene la Real Mina de Guancavelica, 1726.

22. Dos años después del fin del mandato virreinal, Pedro Ambrosio de Bilbao, ex-corregidor de la provincia de Pacajes, pidió al príncipe de Santobuono que siguiera apoyando el proyecto de abolición de la mita. ASN, Archivio privato Santobuono, vol. 35, f. 1, Pedro Ambrosio de Bilbao al príncipe di Santo Buono, La Paz, 25 mayo 1722.

23. PeARCE, 2014: 108. «Abolition of the mita, meanwhile, was simply ignored in Huancavelica, the superintendent writing five years later to explain his motives for not bringing it into effect. The Crown made no attempt to pursue the matter, and a royal decree of 1733 then officially «restored» the Huancavelica labor draft».

24. Sobre los proyectos, no aprobados, relacionados con el monopolio del tabaco y el papel cfr., AGI, Audiencia de Lima, legajo 410. Decreto de Felipe $V$ al consejo de Indias, Madrid 6 diciembre 1719. La orden regia era del tenor siguiente: «Remito al Consejo de Indias el projecto adjunto que ha embiado el virrey príncipe de Santo Buono sobre extablecer estancos del tabaco, y papel blanco en el Perú, para que en su vista me consulte le que se le ofreciere y pareciere». 
de los indígenas empleados en las minas de Huencavelica; la reducción del precio del azogue; el aumento del Quinto Real y los ingresos de los tributos de los indios ${ }^{25}$.

Si el primer aspecto no requería ningún análisis específico, para los otros tres fue necesaria la elaboración de una memoria detallada. Tras la reelaboración de algunas informaciones remitidas por el cardenal Alberoni, Caracciolo había calculado que el uso en Perú de Io ooo quintales de mercurio de España implicaría un gasto de 132 ooo pesos: 50 ooo para extracción, 20 ooo para el transporte de Cádiz a Portobelo, I7 ooo para el cruce del río Chagre hasta Cruces y de allí, vía terrestre a Panamá, para finalmente sumar 45000 para llegar a Lima y Arica, «de donde debe hacer el Almacén principal para el repartamiento de los minerales» ${ }^{26}$. Respetando el precio de venta presente, 58 pesos y 6 reales el quintal, la Real Hacienda habría obtenido un ingreso neto de 455500 pesos anuales. Además, si se hubieran completado los proyectos para la apertura de la nueva ruta de la Gorgona y el traslado del cacao desde Guayaquil, los gastos de viaje se reducirían a solo 15 ooo pesos y los ingresos se habrían incrementado así a 485500 pesos $^{27}$.

En cuanto al incremento del Quinto Real, que de ordinario nunca había superado los 70 ooo pesos, Caracciolo se basó en la experiencia adquirida por los oficiales reales de Nueva España, donde el uso del mercurio de la península Ibérica había registrado un incremento de 200 ooo pesos. Finalmente, el levantamiento de los indígenas de la obligación de trabajar en las minas de Huencavelica podría garantizar mejores condiciones de vida y contribuir a iniciar una recuperación demográfica con el consiguiente aumento de la recaudación fiscal ${ }^{28}$.

La propuesta hecha por el virrey, según los cálculos realizados para cada punto, beneficiaría a la Hacienda Real con 700 ooo pesos y «esto sobre el logro principal de la grande christiana y piadosa importancia de ocurrir por este medio al alivio de los pobres indios, y a evitar su total destruyción $»^{29}$. Sin embargo, el proyecto, si se implementase, impactaría en primer lugar a los corregidores, que ya no podrían lucrar con el comercio ilícito de mercurio, sino también de otros ministros encargados de su control. El Consejo señaló que el Tribunal de Cuentas de Lima, con papel fechado el 29 de octubre de I7I8, había subrayado un crédito de I 424587 pesos que debía ser cobrado a los mineros y azogueros de Huencavelica. La extinción de la deuda

25. AGI, Audiencia de Lima, vol. 410. Extracto de el proyecto embiado por el príncipe de Santo Bono sobre el transporte de los Azogues de España y la prohivición del trabajo de la mina en Guancavelica. Remitido por S. M. al Consejo con decreto de 2 de diciembre 1719.

26. Ibidem.

27. Gracias a la apertura del camino de la Gorgona, el mercurio se transportaría más fácilmente con carretas. En cuanto al proyecto de cacao, se especificó que se podría contratar el costo del traslado de Panamá a Lima, «respecto de que a la buelta de los navios vacíos de Acapulco podrían pasar a Panamá a cargar los azogues, y solo con la demora de tres meses más de viaje de ida y buelta desde Lima a Arica se lograría el transporte sin más gasto que el de 15 ooo pesos» (ibidem).

28. El príncipe de Santobuono también especificó que no se debería prestar atención a «la objectión que alguno pudiera poner a esta deliberación diciendo que poniéndose en práctica perderá S. M. el beneficio de los quintos del azogue que se sacan de la referida Mina de Guencavelica, porque se consideran los grandes gastos que hace la Real Hacienda de su manutencion la de los sueldos de ministros y los grandes rezagos que los mineros quedan deviendo en cada un año de el dinero efectivo que se les anticipa por la Real Hacienda para el trabajo de la mina (sin el qual no se lograría saca alguna de metal) cuios rezagos o débitos de mineros llegan ya a cerca de un millón y medio» (ibidem).

29. Ibidem. 
podría verse comprometida con la ejecución del proyecto y, en consideración de la entidad de la suma, se creía que el virrey y el fiscal de la Audiencia debían, en primer lugar, trabajar para que la Real Hacienda pudiera cobrar primordialmente el pago.

Junto a estas ideas reformistas del príncipe de Santobuono, un novedoso proyecto de revisión del sistema postal, que se discutió en el Consejo de Indias el 6 de diciembre de I7I9, no se consideró necesario ${ }^{30}$. De hecho, fue Bernardo Tinajero de la Escalera quien expresó una opinión negativa, argumentando que los motivos expuestos por el virrey para redactar la propuesta no tenían fundamento ${ }^{31}$. Caracciolo asumía que el retraso en las comunicaciones entre Madrid y Lima, a veces incluso tres años, era la principal causa

«de los mayores desórdenes que allá se experimentan, así en lo que mira a los intereses de S. M. y buena administración de justicia a aquellos vassallos, como en los intereses privados de los mismos vasallos tocante a las cosas de comercio, siendo tal dilación la causa más propria de todas las faltas de los poder havientes y de los encomenderos que no cumplen con la puntualidad que deven con sus principales» ${ }^{32}$.

El napolitano consideraba que la ampliación de los poderes del virrey y de los ministros reales había sido resultado de la falta de cartas proveniente de la Corte, lo que permitió una mayor libertad y arbitrariedad en el ejercicio del gobierno peruano. Al formular su proyecto, Santobuono tuvo en cuenta tres puntos fundamentales: su sostenibilidad en términos económicos; su factibilidad, sin incurrir en excesivas dificultades logísticas; y el cumplimiento del artículo 75 de las instrucciones, que obligaba a los virreyes a enviar una vez al año un despacho con información sobre el estado del reino ${ }^{33}$.

Con respecto al análisis de los gastos anuales, Caracciolo calculó los siguientes: 3 ooo escudos de plata para la compra de un paquebote, más otros i ooo para las reparaciones a realizar después de un año de navegación; 4284 escudos para los sueldos de los oficiales a bordo ${ }^{34} ; 2430$ escudos para aprovisionamiento. Los gastos, así calculados, ascendían a Io 7I4 escudos anuales pero, considerando el uso de dos embarcaciones, se alcanzaba la suma de 2I 428 escudos, de los cuales 6 ooo se destinarían a la compra de los navíos y los restantes i6 428 escudos a su

30. RB, ms. II/2875, ff. $251 \mathrm{r}-269 \mathrm{v}$, Proyecto del príncipe de Santo Buono sobre establecer un comercio más frequente de cartas entre la corte y aquella provincia (6 diciembre 1719).

31. Cfr. HILL, 2005.

32. RB, ms. II/2875. Proyecto del príncipe de Santo Buono sobre establecer un comercio más frequente de cartas entre la corte y aquella provincia (6 diciembre 1719), cit.

33. «Por lo que conviene a mi servicio y bien de la causa pública de estos mis reynos y los de las Indias, tener noticias frequentes del estado de aquellas provincias y de lo que necessitan para su conservación y defensa os encargo y mando despachéis cada año un aviso a estos reynos dándome cuenta del estado en que se hallaren aquéllos y su dependencias y negocios, estando advertido que en caso de no executarlo cada año vos y vuestros successores mandaré despachar aviso solicitando estas noticias y que el coste y gastos que causare se descontará del sueldo que gozaren» (ibidem).

34. «Un capitán con 30 escudos al mes, 360; un piloto con 25 escudos al mes, 300; un ayudante de piloto con 20 escudos al mes, 240; un contra maestre ydem, 240; un escribano con 16 escudos, 192; un carpintero con el mismo sueldo, 192; un calafate, 192; un dispensero, 192; doze marineros que algunos entiendan el manejo de artillería a 8 escudos al mes, 1152; un condestable con 12 escudos, 144 ; 15 grumetes a 6 escudos, 1080 » (ibidem). 
mantenimiento. Si se hubiera considerado oportuno eximir a la Real Hacienda del pago de los gastos antes mencionados, se podría estipular un asiento -de diez o doce años- con «persona de Andalucía o de otro parage de España» 35 que se habría beneficiado de la posibilidad de utilizar barcos para el transporte de mercancías.

Una vez finalizado el análisis de los gastos, el príncipe de Santobuono presentó la logística del viaje: una embarcación saldría de Cádiz el i de enero para llegar a Portobelo el I4 de febrero y partiría hacia La Habana el 2 de marzo. Desde el puerto cubano reanudaría la navegación el día 20 del mismo mes para desembarcar en Cádiz el 8 de mayo, completando así la travesía circular en i28 días. En cambio, la salida del segundo barco de Cádiz estaba programada para el 24 de marzo y, siguiendo la ruta y el horario del anterior, regresaría el 28 de junio ${ }^{36}$. La propuesta, tan detallada en la descripción de lugares, días y horas, habría traído, según Caracciolo, una indudable ventaja

«demás del alivio que se tendrá de que cada 92 días pueda haver cartas de Lima en Madrid y en el mismo tiempo, con corta diferencia, cartas de Madrid en Lima, se podrá lograr otra más grande conveniencia de abrir un comercio regular de cartas con los mismos dos pacatebotes quatro veces al año con la Nueva España; pues al tiempo que dichos pacatebotes deven llegar a La Habana (...) se podrán hallar en aquella isla las cartas de México dando órdenes S. M. a quel virrey para que tenga prevenido embarcaciones en La Habana en los referidos tiempos para que reciva las cartas ${ }^{37}$.

Bernardo Tinajero no era de la misma opinión: un plan tan preciso de los tiempos de viaje no tomaba en cuenta que «en los accidentes de la mar, no hay juicio humano» y que cualquier pronóstico podría ser fácilmente contradicho por elementos atmosféricos adversos o por ralentizaciones en la navegación debido a los ataques de los corsarios. Además, la hipótesis de otorgar a los asentistas la posibilidad de utilizar paquebotes para el transporte de mercancías contravenía las disposiciones reales, según las cuales ningún buque postal podía cargar mercancías privadas. El ministro no dudó, por tanto, en formular una opinión decididamente opuesta:

«Por todo lo qual, mi sentir es que se deve despreciar la proposición absolutamente, previniéndole al príncipe no entra S. M. en ella, y explicándole que el punto de la istrucción que cita mira a que su celo y cuidado se aplique a remitir continuadamente todos los pliegos del real servicio y de particulares que trafican de Paita y El Callao a Panamá, a cuyo presidente y audiencia encargará los remitan prontamente a Portovelo para que desde allí los dirijan a estos reynos o ya en los navíos de nuevo asiento de negros o en los navíos de aviso de España»38.

35. Ibidem.

36. Ambos buques realizarían dos viajes al año. Los despachos entregados a los oficiales de Portobelo serían, a continuación, enviados a Lima, siguiendo una ruta terrestre de Portobelo a Panamá y otra marítima de Panamá a Lima, con «una embarcación ligera capaz solo para los víveres del correo y marineros». Las comunicaciones de Cádiz llegaban a Madrid por una ruta extraordinaria (ibidem).

37. Ibidem.

38. Ibidem. 
Para el príncipe de Santobuono, la reelaboración de las disposiciones contenidas en las cédulas o en las ordenanzas podría ser percibido como un intento de integrar, también a través de la práctica, un corpus de leyes que de otro modo se habría mantenido insensible a los cambios sociales, políticos y económicos del reino. Respetando la autoridad real y su patrimonio, el virrey consideró posible, por tanto, hacer propuestas que cambiaran el marco regulatorio. Esta creencia surgía, asimismo, en el proyecto relativo al tributo de la alcabala ${ }^{39}$. También en este caso se plantearon de forma detallada y oportuna los objetivos marcados y los caminos a seguir para alcanzarlos.

La recaudación de la alcabala peruana era asignada a individuos a través de una subasta pública. Aunque, tanto para el impuesto como para cualquier actividad comercial se prohibió la participación de los corregidores, a menudo ocultaban su participación detrás de testaferros. Ante la imposibilidad de lidiar con este fenómeno, profundamente arraigado en los territorios del virreinato andino, y en la creencia de que podría aumentar los ingresos anuales en 50 ooo pesos, el príncipe de Santobuono propuso abolir tanto el uso de la subasta pública para confiar en terceros de la colección, como la prohibición impuesta a los corregidores para llevar a cabo prácticas comerciales.

Sobre el primer punto, la reforma incluía la redacción de una lista que contenía los nombres de todos aquellos que tendrían que pagar el derecho de las alcabalas y la provisión del importe de la recaudación. Calculado la hipotética suma a pagar a la Real Hacienda, los corregidores habrían pagado un tercio con el fin de adquirir, mediante pago, el derecho a vender e intercambiar bienes en las provincias de su gobierno. De hecho, el príncipe de Santobuono propuso la normalización de una práctica considerada ilícita en la creencia de que esta medida beneficiaría tanto a la Real Hacienda -a la cual se garantizaban ingresos ciertos y puntuales- como a resolver las tensiones y abusos entre los corregidores y los indios.

En primer lugar, Caracciolo consideró que la violación de la ley por parte de los corregidores era ineludible: los salarios recibidos eran insuficientes para garantizar su sustento y, por lo tanto, se veían obligados a recurrir a trucos ilícitos para obtener mayores ganancias. Además, la insuficiencia del salario pagado había hecho el cargo poco atractivo «con que faltando personas que ocupasen tales empleos de corregidores, cessaría enteramente el curso de la justicia y el desorden sería sin proporción mayor de lo que se supone pueda resultar del comercio de los corregidores» ${ }^{40}$. En segundo lugar, la posibilidad de los oficiales de comerciar en sus respectivas provincias habría ayudado a mejorar las condiciones de los indios, que podrían comprar más fácilmente lo necesario para vestir y llevar a cabo sus actividades laborales ${ }^{41}$.

39. AGI, Audiencia de Lima, legajo 410. «Remito al Consejo de Indias el proyecto adjunto que ha embiado el virrey príncipe de Santobuono sobre el augmento del ramo de alcabalas sin perjuicio de mis vasallos, para que en vista de su contenido me consulte lo que se le ofreciere y pareciere». Decreto de Felipe $V$ al Consejo de Indias, Madrid, 2 diciembre 1719. La alcabala era un impuesto establecido por Alfonso XI, a mediados del siglo XIV, calculado sobre un porcentaje del valor de los bienes vendidos o intercambiados.

40. Ibidem.

41. Caracciolo utiliza una descripción arquetípica de los indios como perezosos e inactivos: «Pues siendo el natural de indio tan torpe y perezoso como se save, que sin la fuerza ni travaxará, ni se vestirá ni pagará lo que deve, ni aun tomará un medicamento para beneficio de su salud si no se le obliga con violencia, no repartiendo 
A la luz de lo expuesto, la propuesta de Caracciolo se articuló esquemáticamente en torno a los siguientes puntos: revocar la ley que obligaba a los corregidores a renunciar a mantener prácticas comerciales; permitirles un «moderado y justificado comercio» de necesidades básicas para los nativos -como «paños, ropa de la tierra, lenzería, cera, herramientas, mulas»- pero prohibir la venta de productos considerados «perjudiciales, como son aguardiente, y a los que son para ellos inútiles como son géneros ricos, sedas, encajes, telas y otros que solo sirven al adorno de las personas y no a la necessidad $»^{42} ; \mathrm{y}$, por último, asignar a los corregidores el pago de un tercio del valor de la alcabala que se requiera en las provincias bajo su mando, a fin de garantizar a la Real Hacienda un ingreso seguro.

Si los anteriores proyectos incluían reformas destinadas a incrementar los ingresos en las Real Hacienda, la nueva planta de la alcabala del Perú se atenía, en cambio, a la revisión del sistema administrativo mediante la supresión de los problemas derivados de los retrasos en la recaudación de impuestos y de pago de deudas ${ }^{43}$. El elemento principal de la reforma consistía en una redistribución de funciones entre los ministros de la Real Hacienda y en la designación, en el interior de la Real Audiencia, de un tercer fiscal que se sumaría a los dos presentes para los poderes civil y penal, teniendo en este caso una jurisdicción exclusiva dentro del Tribunal de Cuentas.

La opinión formulada por el Consejo de Indias parece pronosticar las acusaciones que convergerían, poco después, en el proceso de residencia contra Santobuono. Así, el príncipe fue acusado de haber presentado un proyecto «tan desnudo que solo se funda en su juicio, quando para tomar su acertada resolución debiera venir vestido de diligencias e instrumentos que calificasen los deshórdenes que se debían enmendar» ${ }^{44}$, amén de haber ignorado aquellas disposiciones reales que hubieran permitido el restablecimiento de la correcta administración hacendística del virreinato. Los miembros del Consejo madrileño inquirieron al virrey las acciones que habría tomado hasta ese momento y qué resistencias había encontrado entre las elites gubernativas y económicas del Perú. Además, invitaron al virrey a consultar nuevamente la respuesta que diese a un despacho real que le fue enviado en septiembre de i7i6: con ello podría rastrear todos los remedios que frenasen el mal funcionamiento de la máquina administrativa y gobernar sin tener que recurrir a «remedios tan extraños como los que contienen su projecto» ${ }^{45}$. En definitiva, los problemas de gestión de la Real Hacienda podrían resolverse mediante un respeto más cuidadoso de las reglas y ordenanzas por parte de los ministros, sin recurrirse, necesariamente, a la implementación de una nueva reforma.

\footnotetext{
y fiando entre ellos los corregidores los géneros de que necessitan para vestirse y para travaxar, como son mulas y herramientas, se quedaran desnudos y en ozio todo el día y, por consequencia, incapaces de pagar los reales tributos» (ibidem).

43. AGI, Audiencia de Lima, legajo 410. Proyecto para la puntual y exacta revissión de las quentas de las caxas del reyno y para la buena administración de sus rentas y de las del tribunal de Cruzada con notable augmento de 100000 pesos cada año en beneficio de la Real Hacienda. Sobre los intentos de reformar la hacienda peruana en la época borbónica, cfr. Céspedes del CAstillo, 1953.

44. Proyecto para la puntual y exacta revissión de las quentas de las caxas del reyno, cit.

45. Ibidem.
} 


\section{NOTA CONCLUSIVA}

La atención que debe prestarse a los procesos reformistas amparados por el virrey Santobuono, y no solo a sus resultados positivos, permite reconstruir procesos menos lineales pero, precisamente por ello, más complejos y profundos. El fracaso de los modelos por Carmine Nicola Caracciolo en ámbitos muy amplios y diferenciados no afecta a la importancia de los caminos tomados y las acciones emprendidas por el pro rex, tanto a nivel local como en las relaciones con Madrid. Es evidente que en cada una de estas propuestas convergían diferentes intereses personales, corporativos o institucionales. La definición del equilibrio entre múltiples poderes en competencia fue uno de los factores más importantes para asegurar el resultado exitoso de las reformas. Sin embargo, hay que tener en cuenta que muchas propuestas adelantadas en las primeras décadas del siglo XVIII fueron un primer intento de cambiar una organización administrativa considerada inadecuada, una especie de laboratorio e intentos que solo después de una extensa revisión y experimentación vieron, en la mitad del siglo, resultados más duraderos. 


\section{BIBLIOGRAFÍA}

Alabrús Iglesias, Rosa María, «La trayectoria política del cardenal Giulio Alberoni (I7o8I720)», Revista de historia moderna, 29 (201I): I7I-I84.

AndúJar CASTILlo, Francisco, «Entre la corte y la guerra. Militares italianos al servicio de España en el siglo XVIII», en Paola Bianchi, Davi Maffi y Enrico Stumpo (coord.), Italiani al servizio straniero in età moderna, Annali di storia militare europea, Milán, FrancoAngeli, 2008: I05-I34.

BARrio Gozalo, Maximiliano, «El Cardenal Alberoni y España: politica religiosa y carrera eclesiástica», Hispania Sacra, I27 (20II): 205-234.

Becker, Jerónimo y Rivas Groot, José María, El Nuevo Reino de Granada en el Siglo XVIII, Madrid, Imp. del Asilo de Huérfanos del Sagrado Corazón de Jesús, I92I.

Crespo Solana, Ana, La Casa de Contratación y la Intendencia General de la Marina en Cádiz (I7I7-I730), Cádiz, Universidad de Cádiz, I996.

CÉSPedes del CAstillo, Guillermo, «Reorganizacion de la Hacienda virreinal peruana en el siglo XVIII», Anuario de historia del derecho español, 23 (I953): 329-370.

Eissa-BArroso, Francisco A., «El Abate, el Consejo y el virreinato: la política cortesana y la primera creación del virreinato de Nueva Granada (I7I7-I723)», en Francisco Fernández Beltrán y Lucía Casajús (eds.), España y América en el Bicentenario de las Independencias. I Foro Editorial de Estudios Hispánicos y Americanistas, Las Palmas, Universidad de Las Palmas de Gran Canaria, 2012: 293-3I4.

Eissa-Barroso, Francisco A., The Spanish Monarchy and the Creation of the Viceroyalty of New Granada (I7I7-I739), Leiden, Brill, 2016.

FAvarò, Valentina, «Tra Roma e Venezia. L'attività diplomatica di Carmine Nicola Caracciolo negli anni della Guerra di Successione Spagnola», Cheiron, I (2018): IIO-I33.

Favarò, Valentina, Pratiche negoziali e reti di potere. Carmine Nicola Caracciolo tra Europa e America (I696-I725), Soveria Mannelli, Rubbettino, 2019.

Fragnito, Gigliola (coord.), Elisabetta Farnese principessa di Parma e regina di Spagna, Roma, Viella, 2009.

Hill, Ruth, Hierarchy, Commerce, and fraud in Bourbon Spanish America: A postal Inspector's exposé, Nashville, Vanderbilt University Press, 2005.

Kuethe, Allan J., "Cardinal Alberoni and Reform in the American Empire», en Francisco A. Eissa-Barroso y Ainara Vázquez Varela (coord.), Early Bourbon Spanish America. Politics and Society in a Forgotten era (I700-I759), Leiden, Brill, 2013: 23-38.

López-Cordón, María Victoria, «La evolución de las damas entre los siglos XVII y XVIII», en José Martínez Millán y Maria Paula Marçal Lourenço (coord.), Las relaciones discretas entre las Monarquías Hispana y Portuguesa: las Casas de las Reinas (siglos XV-XIX), Madrid, Ediciones Polifemo, 2008: I357-I398.

MAFrici, Mirella, Elisabetta Farnese. Una regina italiana nella grande politica europea, Roma, Avagliano, 20I9.

Maqueda Abreu, Consuelo, Alberoni, entorno jurídico de un poder singular, Madrid, UNED, 2009.

Martínez Navas, Isabel, «Alberoni y el gobierno de la Monarquía española», Revista electrónica del Departamento de Derecho de la Universidad de la Rioja, 8 (2010): 63-IIo. 
Meza Villalobos, Néstor, «Felipe $V$ y el problema etico politico de la provision de mano de obra a la mineria del Perù y Nuevo Reyno de Granada», Estudios sobre politica indigenista espanola en America, 2 (1976): 313-343.

Pearce, Adrian J., The Origins of Bourbon Reform in Spanish South America, I700-I763, New York, Palgrave Macmillan, 2014.

Puente BRUnKe, José de la, «Las estrellas solo lucen cuando el sol se pone. Los ministros de la audiencia de Lima en el siglo XVIl y sus expectativas», Illes $i$ imperis, I4 (20I2): 49-68.

Sodano, Giulio, «L'occhio della madre. La politica internazionale di Elisabetta Farnese», en Rosanna Cioffi, Luigi Mascilli Migliorini, Aurelio Musi, Anna Maria Rao (coord.), Le vite di Carlo di Borbone. Napoli, Spagna e America, Napoli, Arte'm, 20II: 8I-9I.

TAU ANZOTAEGUi, Victor, «Las reformas borbonicas y la creacion de los nuevos virreinatos», en Feliciano Barrios Pintado (ed.), Congreso internacional. El gobierno de un mundo. Virreinatos y audiencias en la América Hispánica, Cuenca, Universidad de Castilla-La Mancha/Fundación del Pino, 2004: 43I-445.

Vázquez Gestal, Pablo, Una nueva majestad. Felipe V, Isabel de Farnesio y la identidad de la monarquía (I700-I729), Madrid, Marcial Pons Historia, 2013. 



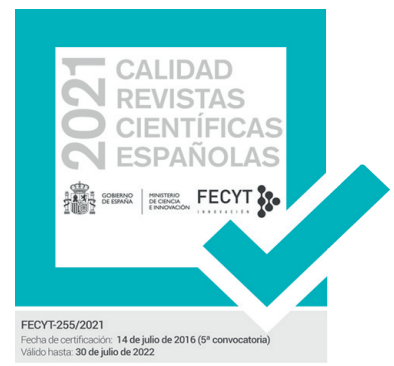

SERIE IV HISTORIA MODERNA

REVISTA DE LA FACULTAD DE GEOGRAFÍA E HISTORIA

AÑO 2021

ISSN: 1131-768X

E-ISSN 2340-1400

\section{4 \\ 西 ESPACIO, TIEMPO Y FORMA}

Monográfico - Special Issue: La política ultramarina de las monarquías ibéricas (circa 1700-1750): una historia de fracasos y éxitos relativos The Overseas Policy of the Iberian Monarchies (Circa 1700-1750): A History of Failures and Relative Successes

15 Roberto Quirós Rosado y MARIA FERnANDA BICALHO La política ultramarina de las monarquías ibéricas (circa 1700-1750): una historia de fracasos y éxitos relativos / The Overseas Policy of the Iberian Monarchies (Circa 1700-1750): A History of Failures and Relative Successes

\section{Guillaume Hanotin}

Defender negocios en tiempo de convulsión política: las elites mercantiles francesas durante la guerra de Sucesión española / Protecting Business in Time of Crisis: French Trademen during the War of Spanish Succession

\subsection{Maria Fernanda Bicalmo}

Ultramarino y el auge de los secretarios de Estado en Portugal durante la primera mitad del siglo XVIII / Sobre este modo de resolver e despachar os negócios. The decline of the Overseas Council and the Rise of the Secretaries of State in Portugal during the First Half of the $18^{\text {th }}$ Century

\section{9}

\section{VALENTINA FAVARò}

El fracaso de los proyectos de reforma en el virreinato peruano de principios

del siglo XVIII. Las propuestas de Carmine Nicola Caracciolo, príncipe de Santobuono the Eighteenth Century. The Proposals of Carmine Nicola Caracciolo, Prince of Santobuono

\section{7}

\section{ROBERTO QUIRÓS ROSADO}

Ecos de un mercantilismo truncado. El conde de Pinos Puente y la diplomacia comercial de Carlos VI en la corte de Lisboa (1723-1724) / Echoes of a Failed Mercantilism. The Count of Pinos Puente and the Commercial Diplomacy of Charles VI at the Court Of Lisbon (1723-1724)

\section{Junia Ferreira Furtado}

Portuguese America under Foreign Threat and the Creation of the Concept of uti possidetis in the First Half of the $18^{\text {th }}$ Century / La américa portuguesa bajo la amenaza exterior y la creación del concepto de uti possidetis en la primera mitad del siglo XVIII

\section{Miscelánea $\cdot$ Miscellany}

\section{José Antonio Mateos Royo}

con Cataluña / Trade Policy and Monetary Circulation in Aragon: Conflicts and Agreements with Catalonia (1535-1565)

\section{Fernando Altoé}

panegíricos atribuidos a la impresion. Un estudio de la trayectoria de dos the Trajectory of Two Panegyrics Attributed to João de Barros

\section{Francisco Velasco Hernández}

reino de Murcia (siglos XVI y XVII) / The Influence of the Berber Corsican on the Late Repopulation on the Coastal Area on the Kingdom of Murcia (XVI and XVII Centuries)

\section{José Antonio Martínez Martínez}

Criados, jornaleros y esclavos al servicio de la familia: la servidumbre de Serfdom of the Muñoz de Otálora in the $17^{\text {th }}$ Century

\section{Víctor Daniel Regalado González-Serna}

Benito de Medina a raíz de su ingreso en el cabildo catedral de Sevilla en 1669 / «Not a Single Good Portuguese». Accusations against Priest Alonso Benito de Medina when Entering the Cathedral Chapter of Seville in 1669

\section{José Herrera Reviriego}

organigrama comercial y militar de la Gober a tólo mitad del siglo XVII / "Only Time will Tell us»: The Role of Taiwan within the Commercial and Military Organization of the Philippine's Governoration during the First Half of the Seventeenth Century

\section{Manuel-Reyes García Hurtado}

de Rande, 1719-1733 / Vicissitudes of the Rescue Companies of the Sunken Ships in the Battle of Rande, 1719-1733

\subsection{Marcos de Miguel Muñoz}

Caballeros in 1769 


\section{4 ESPACIO, TIEMPO Y FORMA}

\section{Javier Tinoco Domínguez}

Tensiones sociopolíticas en el marco del catastro de Ensenada en Jerez de la Frontera: estudio de un conflicto institucional / Socio-Political Tensions within the Framework of Cadastre of Ensenada In Jerez de la Frontera: A Studying of an Institucional Conflict

\section{Pablo Fernández Albaladejo}

Fábulas de origen y gramática de nación en la España del siglo XVIII. A propósito de algunos trabajos de Francisco Martínez Marina / Origin's Fables and Grammar of Nation in the XVIII Century Spain. About some Works by Francisco Martínez Marina

\subsection{José María IÑURRITEgui Rodríguez}

Constitución increada: Francisco Martínez Marina y la crítica bíblica / Uncreated Constitution. Francisco Martínez Marina and Biblical Criticism

\subsection{David A. Abián Cubillo}

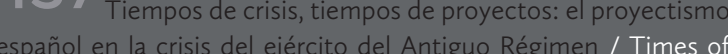
crisis, Times of Projects: The Spanish proyectismo during the Army's Crisis in the Ancient Regime

Taller de historiografía · Historiography Workshop

\section{Ensayos · Essays}

\subsection{ChrISTOPH ROSENMÜLLER}

«Tan peligrosas y feas conspiraciones»: la relación escrita por el embajador austriaco Christoph Migazzi en 1754 sobre la caída del marqués de la Ensenada / «Dangerous and Ugly Conspiracies». The Report of the Austrian Ambassador Christoph Migazzi on the Fall of the Marquis of la Ensenada in 1754

\subsection{Serge Gruzinskı}

Quelle histoire enseigner en 2021 ? / ¿Qué historia enseñar en 2021?

\subsection{Carlos Amate Pizarro}

Las relaciones hispano-chinas en el siglo XVI: síntesis e interpretación a la luz de la reciente historiografía / The Hispanic-chinese Relationship in the XVI Century: Synthesis and Interpretation in the Light of Recent Historiography

\section{Reseñas • Book Review}

521 Bolufer Peruga, Mónica, Arte y artificio de la vida en común. Los modelos de comportamiento y sus tensiones en el Siglo de las Luces, (Julio ArRoyo Vozmediano) 


\section{4 ESPACIO, TIEMPO Y FORMA}

525 Melón, Amando, Alejandro de Humboldt. Vida y obra (Carlos Martínez Shaw)

52 Commentary to Tatiana Seijas' review of The Atlantic World and the Manila Galleons: Circulation, Market, and Consumption of Asian Goods in the Spanish Empire (JosÉ LuIs GASCH TOMAS)

533 Braguier, Laurey, Servantes de dieu. Les beatas de la Couronne de Castille (1450-1600) (Manuela Águeda GARCÍA-GARRIDO)

539 Romeo, María CRuz; SAlomón, María Pilar; TABANERA, Nuria (eds.): Católicos, Reaccionarios y Nacionalistas. Política e identidad nacional en Europa y América Latina Contemporáneas (JAVIER M. Dos SANTOS)

54 Heredia López, Alfonso Jesús, El control de la corrupción en la Monarquía Hispánica. La Casa de la Contratación (1642-1660) (José Manuel Díaz Blanco)

17 Andújar Castillo, Francisco, El Atila de Madrid. La forja de un banquero en la crisis de la monarquía (1685-1715) (Aitor Díaz PAREdes)

551 Serrano Aviles, Javier y Mojarro, Jorge (eds.) Prada GonzALEZ, María (coord. de ilustraciones), En el archipiélago de la Especiería. España y Molucas en los siglos XVI y XVII (İ̃̃ıGo VALPUESTA VILLA)

555 Díaz Ceballos, Jorge, Poder compartido. Repúblicas urbanas, Monarquía y conversación en Castilla del Oro, 1508-1573 (JUAN SEbAstián Gómez GonZÁleZ)

51 Edelmayer, Friedrich, Massimiliano II, Filippo II I'Italia imperiale. II marchesato di Finale, i diritti imperiali e il «camino spagnolo» (RAFAEL VALLADARES)

56 Escribano-PÁEZ, José M., Juan Rena and the Frontiers of Spanish Empire, 1500-1540 (DAvid Martín Marcos)

56 ARnOLD, David, La Era de los Descubrimientos (1400-1600), Madrid, Alianza Editorial, 2021, 184 Pp., ISBN: 978-841362-172-2 (CARlos Amate Pizarro) 